\title{
Riesgo de enfermedad tuberculosa en pacientes con artritis reumatoide
}

\author{
Rocío Gamboa ${ }^{1}$, Eduardo Acevedo ${ }^{1}$, César Gutiérrez ${ }^{2}$, Darío Ponce de León ${ }^{3}$, \\ César Pastor ${ }^{1}$, José Alfaro ${ }^{1}$, Mariano Cucho ${ }^{1}$, Alfredo Sánchez ${ }^{1}$, Risto Perich ${ }^{1}$, \\ César Sánchez ${ }^{1}$
}

Resumen

Palabras clave

\begin{abstract}
Antecedentes: Los estudios sobre riesgo de enfermedad tuberculosa (ET) en artritis reumatoide(AR) en pacientes no usuarios de terapia biológica son controversiales. Objetivo: Evaluar AR como factor de riesgo independiente para ET. Diseño: Estudio de cohortes no concurrente. Lugar: Red asistencial Hospital Guillermo Almenara Irigoyen, Lima-Perú, hospital docente. Participantes: Pacientes con AR no usuarios de terapia biológica. Intervenciones: Una cohorte de pacientes con AR no usuarios de terapia biológica (cumplimiento de criterios 1987 del ACR) fue pareada por edad y género con una cohorte control (pacientes con trastornos no inmunológicos). Se realizó una entrevista personal. El seguimiento para la cohorte AR se inició en el diagnostico de AR y culminó en el momento de la entrevista o en el diagnóstico de ET(los controles fueron seguidos el mismo periodo de tiempo). Se calculó la densidad de incidencia (DI) para ET en cada cohorte y el riesgo relativo (RR). La probabilidad de ET de acuerdo al tiempo de enfermedad fue comparada mediante curvas de Kaplan Meier. Se aplicó un modelo de Cox para ajustar drogas y patologías predisponentes (hazard ratio HR). Principales medidas de resultados: Enfermedad tuberculosa en pacientes con artritis reumatoide. Resultados: La cohorte AR y los controles (667 y 664 pacientes, respectivamente) tuvieron 6940,75 y6666,53 personas-año de seguimiento. La edad al diagnóstico de AR fue 46,65 años. Un 29,7\% de 195 pacientes AR fueron PPD positivos. Quince pacientes AR yocho controles desarrollaron ET(DI de 216,1/100000 y 122,1/100 000 personas/año). El RRpara TBC fue 1,8(IC 95\% = 0,8-4,2) yluego del ajuste para drogas y comorbilidades predisponentes de ET, el HR fue 1,69(IC 95\%=0,26-10,93). No hubo diferencia al comparar las curvas de Kaplan Meier $(p=0,19)$. Conclusiones: La incidencia de ET entre los pacientes con AR fue mayor que en los pacientes sin dicha condición; sin embargo, esta diferencia no fue significativa luego de ajustar variables confusoras.
\end{abstract}

Artritis reumatoide; tuberculosis; estudio de cohortes; factores de riesgo.
Tuberculosis risk in patients with rheumatoid arthritis

\section{Abstract}

Background: Studies on tuberculosis (TB) risk in patients with rheumatoid arthritis (RA) non-users of biological therapy show contradictory results. Objetives: To determine RA as independent risk factor for TB. Design: Non concurrent cohort study. Setting: Guillermo Almenara Asistential Net, Lima-Peru, a teaching hospital. Participants: Biological therapy non-users RA patients. Interventions: RA patients fulfilled the ARA1987 diagnosis criteria

1 Servicio de Reumatología, Hospital Guillermo Almenara Irigoyen. LimaPerú.

2 Sección de Epidemiología, Instituto de Medicina Tropical DanielA. Carrión, Universidad Nacional Mayor de San Marcos. Lima-Perú.

3 Servicio de Medicina Interna, Hospital Guillermo Almenara Irigoyen. LimaPerú. and were biological therapynon-users. Control group was paired by age and sex to RA patients. Patients in control groups had non immunological disorders. Clinical information was completed by interviews. RA patients follow-up was started at the time of diagnosis and ended at the time of interview and/or TB diagnosis. Density incidence (DI) was found for each cohort and TB relative risk(RR) was calculated. To evaluate time length to TBevolution a Kaplan Meier curve was graphed and compared both groups with log-rank test. Drugs and predisposing TB pathologies were analyzed. Main outcome measures: Tuberculosis in patients with rheumatoid arthritis. Results: Six hundred and seventy six out of 808 RA patients and 664 controls qualified for inclusion criteria's. $R A$ and control groups reached 6940,75 and 6666,53 follow-up patients-year, respectively. RA cohort mean age was 46,65 at RA diagnosis. Only $29,7 \%$ of RA patients had a positive tuberculin reaction. Fifteen TB cases were identified in the RA cohort and 8 at the control group, yielding a mean DI of 216,1/100000 and 122,1/ 
100000 patients-year respectively. TBRR was 1,8(IC 95\%=0,84,2), and after adjusting drugs and co-morbidity the HR was 1,69 (IC 95\%=0,26-10,93). Statistically significant difference was not found with Kaplan Meier curves comparison $(p=0,19)$. Conclusions: We did not find a higher risk of rheumatoid arthritis patients to develop tuberculosis

Key words: Arthritis, rheumatoid; tuberculosis; cohort studies; risk factors.

\section{INTRODUCCIÓN}

La artritis reumatoide (AR) tiene una incidencia y prevalencia variable a nivel mundial ${ }^{(1-3)}$. En países en vías de desarrollo, la prevalencia de pacientes con AR es menor al $0,5 \%\left({ }^{4}\right)$. Se estima que al menos un $50 \%$ de estos pacientes sufrirá un episodio infeccioso durante su enfermedad $\left({ }^{5,6}\right)$, condicionando un aumento de la mortalidad $\left({ }^{7}\right)$. Doran y col. encuentran que los pacientes con AR tienen $88 \%$ más probabilidad de sufrir una infección respiratoria baja en relación a controles poblacionales ${ }^{8}$ ). De todas las infecciones, el problema de la tuberculosis (TBC) en pacientes con AR ha ganado la atención de los reumatólogos a partir de su incremento luego de la introducción de los antagonistas del factor de necrosis tumoral alfa (FNT-alfa) ${ }^{(9-14}$ ); determinándose ciertas reglas en relación a la seguridad de su administración $\left({ }^{15,16}\right)$. Sin embargo; la tasa de incidencia de TBC en pacientes con AR sin uso de terapia biológica es hasta el momento controvertida. Pocos estudios han investigado esta incidencia $\left({ }^{17-19}\right)$.

En lo países en vías de desarrollo, AR es una enfermedad que no recibe prioridad en las políticas de salud frente a otras enfermedades prevalentes, como la misma enfermedad tuberculosa. El Perú es considerado, por otro lado, un país endémico de TBC, con una tasa de incidencia, para el año 2000 , de $133,9 / 100000\left({ }^{20}\right)$. La problemática de enfermedad tuberculosa entre los pacientes AR en realidades como la nuestra cobra por ello gran importancia. Ante la ausencia de estudios que evalúen la asociación de TBC y AR en pacientes no usuarios de terapia biológica, en un país endémico para TBC, es que el presente estudio tuvo por objetivo analizar la AR como factor de riesgo independiente para tuberculosis y determinar la densidad de incidencia (DI) de TBC en una cohorte de pacientes con AR en el Hospital Nacional Guillermo Almenara (HNGAI) y compararla con un grupo control.

\section{MATERIALES Y MÉTODOS}

Se realizó un estudio de cohortes no concurrente. Se seleccionó pacientes que formarían parte de las cohortes entre aquellos atendidos en el servicio de Reumatología del HNGAI, entre enero de 2003 y marzo de 2004. Se investigó luego la historia médica de los dichos pacientes. Se incluyó pacientes que cumplían con los criterios ACR-87 para AR $\left({ }^{21}\right)$ y mayores de 17 años al momento del diagnóstico. Los usuarios de terapia biológica, los pacientes con síndromes de superposición y/o infectados con HIV fueron excluidos. A cada paciente de la cohorte AR se le asignó un control pareado por edad ( \pm 1 año) y género. Los controles fueron atendidos por patologías no inmunológicas (osteoartrosis, osteoporosis, fibromialgia y otras enfermedades). El antecedente personal o el de contacto TBC fue completado con una entrevista personal o telefónica.

Para la cohorte AR, el tiempo de exposición se definió como el periodo transcurrido entre:

a. El diagnóstico de AR y la última atención registrada en la historia clínica, si en el paciente no se documentaba enfermedad tuberculosa (ET).

b. El diagnóstico de AR y el momento del diagnóstico de TBC, si se documentaba dicha enfermedad en la historia. 
El tiempo de seguimiento para los controles fue el mismo que el de sus pares con AR.

En ambas cohortes, se definió la presencia de enfermedad tuberculosa por baciloscopia positiva, cultivo positivo y/o anatomía patológica compatible con TBC (granuloma caseoso). Se analizó las siguientes variables relacionadas con riesgo de desarrollar TBC: a) comorbilidad predisponente, como diabetes mellitus (DM), insuficiencia renal crónica (IRC), neoplasias hematológicas o sólidas (NM), enfermedad pulmonar crónica (EPOC) y hemofilia; b) terapia inmunosupresora, como prednisona (PDN) en una dosis mayor a $15 \mathrm{mg} / \mathrm{d}$ por un año o más de $700 \mathrm{mg}$ / año acumulado (a lo largo de la enfermedad); el uso de metotrexato (MTX), azatioprina (AZT), ciclofosfamida (CFA), ciclosporina (CP) y leflunomida (LEF) en cualquier dosis también fue analizado; c) contacto epidemiológico, intra y extrafamiliar; d) historia de infección tuberculosa, es decir, diagnóstico de TBC previo al de AR.

Se realizó una prueba de tuberculina (PPD) para documentar infección, si el paciente consentía dicho procedimiento. Se consideró positivo un resultado mayor o igual a $5 \mathrm{~mm}$ (en la cohorte AR) o $10 \mathrm{~mm}$ (en la cohorte control), a las 48 horas posteriores a la prueba, según parámetros de clasificación recomendados por la Sociedad Americana del Tórax (SAT) ( ${ }^{22}$ ) y empleados previamente por nuestro grupo $\left({ }^{23}\right)$. Se analizó el uso de isoniacida (INH), como profilaxis para TBC. Todos los pacientes fueron informados sobre la naturaleza del estudio, según normas establecidas $\left({ }^{24}\right)$.

Las características generales de la cohorte fueron representadas mediante promedios y porcentajes. La densidad de incidencia fue calculada dividiendo el número total de eventos (enfermedad tuberculosa) entre el total de años-persona de seguimiento. Se calculó el riesgo relativo (RR) para desarrollar TBC y se elaboró curvas de KaplanMeier para determinar la probabilidad de desarrollar TBC posterior al diagnóstico de AR o para el tiempo de observación en los pacientes del grupo control. Se comparó ambas curvas mediante la prueba del rango logarítmico (log-rank). Se analizó factores de riesgo asociados a TBC, mediante análisis bivariado. Un modelo de regresión de Cox fue construido para determinar el hazard ratio ajustado (HR) de cada variable y analizar AR como factor de riesgo. El nivel de significación para todas las pruebas de hipótesis fue 0,05.

\section{RESULTADOS}

De 808 historias clínicas revisadas, 676 pacientes cumplieron con los criterios de inclusión. Se seleccionó 664 controles. El tiempo de seguimiento en cada grupo fue 6940,75 y 6666,53 personas-año, respectivamente. El seguimiento promedio fue 10,30 años. La edad promedio de la cohorte AR al diagnóstico fue 46,6 años y la relación mujer/varón fue 6,7. No se encontró diferencia significativa al comparar ambas cohortes según la edad $(57,21$ vs. 57,37 años; $p=0,82)$ y género $(87,1 \%$ vs. $87,6 \%$ de mujeres, $p=0,79)$. Los fármacos analizados más utilizados por la cohorte AR fueron PDN y MTX.

El $27,7 \%$ de la cohorte AR presentó un contacto epidemiológico de TBC; la mayoría de éstos fue extrafamiliar (trabajadores de salud). El contacto intrafamiliar correspondió en su mayor parte a familiares de primer grado. Se registró un $10,2 \%$ de antecedente de TBC previo al diagnóstico de AR. En 195 pacientes AR se logró realizar una prueba de tuberculina y $70,3 \%$ tuvo resultado negativo (Figura 1). Un 3\% de la cohorte AR recibió profilaxis con $\mathrm{INH}$; las indicaciones no fueron claramente establecidas en las historias clínicas. 


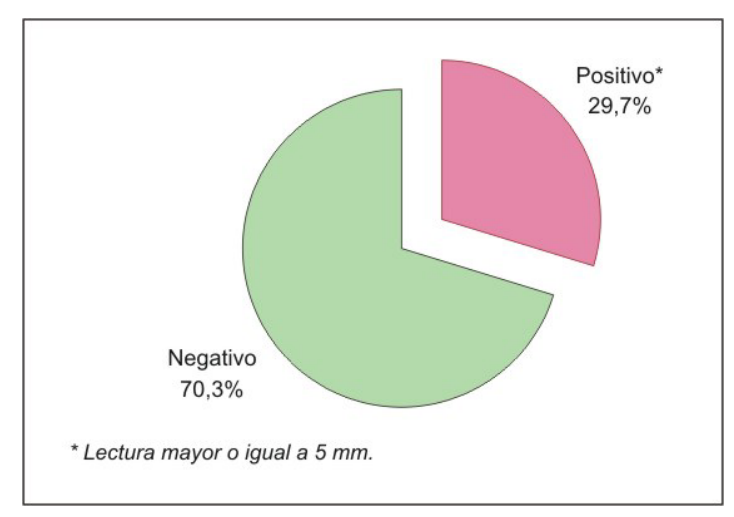

Figura 1. Prueba de PPD en 195 pacientes de la cohorte AR.

Hubo 15 casos de TBC posterior al diagnóstico entre los pacientes con AR, lo que representó una DI de 216,11/100 000 persona-año. El 87,7\% de los casos en este grupo se registró en los primeros 10 años posteriores al diagnóstico. El $66,7 \%$ de los casos tuvo localización pulmonar. En la cohorte control se registró 8 casos de TBC, con una DI de 122,08/100 000 personasaño. Ninguno de los casos de TBC recibió profilaxis y tres de ellos tuvo contacto epidemiológico intrafamiliar. No se registró muertes. En la cohorte AR, la probabi-

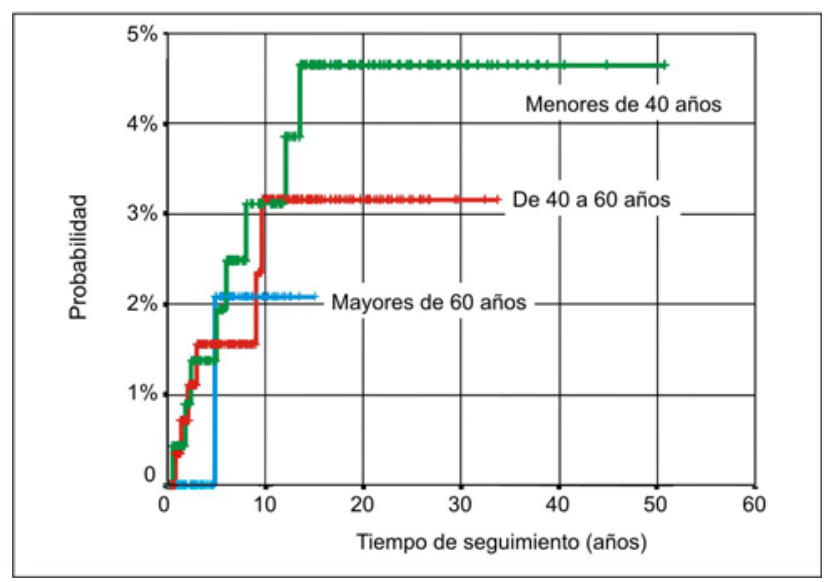

Figura 2. Curvas de Kaplan-Meier: probabilidad de enfermedad tuberculosa según grupos etarios en la cohorte AR. lidad para TBC según grupos etarios no evidenció diferencia estadísticamente significativa (valor $p$ para log-rank $=0,74$ ). (Figura 2).

Al comparar la DI de ambas cohortes, se encontró un RR de 1,77 (IC 95\% = 0,75 $4,17)$. No hubo diferencias estadísticamente significativas en la probabilidad de desarrollar TBC en el grupo de pacientes con AR comparado al grupo control $(p=0,19$ para la prueba log-rank) Figura 3. Al aplicar el modelo de regresión de Cox, en el cual se incorporó las variables de tratamiento inmunosupresor y comorbilidades, no hubo diferencia entre los pacientes con AR $y$ los controles $(\mathrm{HR}=1,69 ; \quad \mathrm{IC}=95 \%=$ $0,26-10,93)$.

\section{DISCUSIÓN}

La TBC es causa importante de mortalidad por origen infeccioso. América latina es una de las zonas más afectadas por esta enfermedad y el Perú es un país endémico (121,2 por 100000 habitantes para el año 2003). Se estima que, en nuestro país, se presenta un $25 \%$ de los casos registrados en toda Latinoamérica $\left({ }^{25}\right)$. Los pacientes

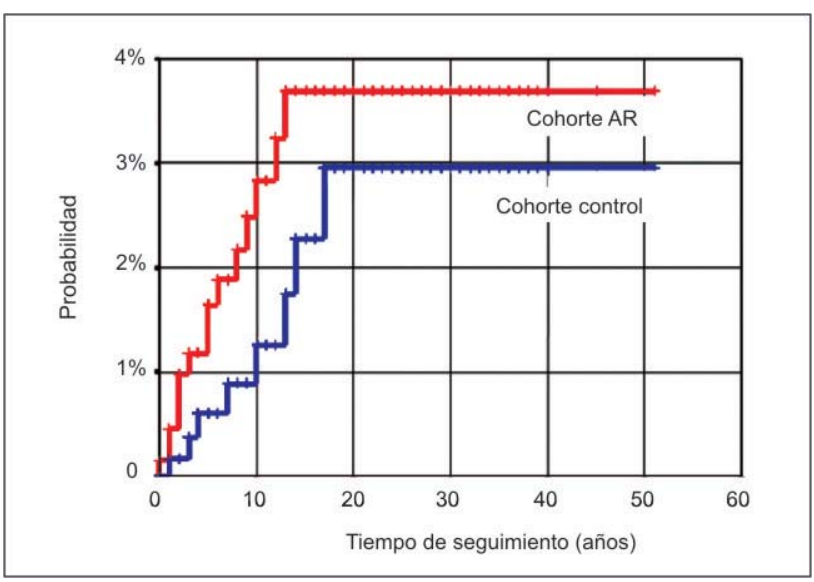

Figura 3. Curva de Kaplan-Meier para el desarrollo de TBC. 
con enfermedades reumatológicas tendrían una alta incidencia de TBC $\left({ }^{5,9,26-29}\right)$. Nuestro grupo encontró una alta prevalencia de enfermedad tuberculosa entre pacientes con LES $\left({ }^{30}\right)$. Sin embargo, hay controversias sobre la incidencia TBC entre pacientes AR sin uso de terapia biológica y los trabajos generados al respecto son difíciles de comparar, por la diferencias en los diseños utilizados. Este es el primer trabajo nacional que indaga la incidencia de $\mathrm{TBC}$ en una cohorte de pacientes con AR.

Nosotros encontramos que hay una tendencia a riesgo elevado de padecer enfermedad tuberculosa entre pacientes con AR, pero que no fue significativa. Nuestro estudio demuestra que, controlando variables confusoras predisponentes para TBC, AR no se comportó como factor de riesgo independiente para desarrollar ET, si bien se documenta una alta incidencia de dicha enfermedad. Por el contrario, en Europa, Carmona y col. $\left({ }^{17}\right)$ encontraron un riesgo para TBC de 4,13 entre los pacientes AR, cuando los compararon con la tasa de infección en la población general, ajustada por edad y género. Sin embargo, las fuentes de información usadas por este grupo fueron diferentes (EMECAR vigila todas las formas de TBC en la población AR; la Red Nacional de Vigilancia Epidemiológica de España solo las formas pulmonar y meníngea). Habría entonces subestimación de la incidencia de TBC en la población general. Contradictoriamente a este estudio, Wolfe y col. $\left({ }^{18}\right)$, en una amplia cohorte norteamericana (10 782 pacientes AR), concluyeron que la DI de TBC entre pacientes AR no estaba incrementada en relación a la población general. No se ajustó tasas, el grupo racial analizado no era acorde con la tasa nacional para comparación y el intervalo de confianza fue muy amplio. La identificación de enfermedad e infección tuberculosa se basó en una encuesta a los sujetos, indagando presencia de PPD positivo o TBC en algún momento de su vida, con lo que se introduciría un sesgo de información. Yoshinaga encontró, por otro lado, una prevalencia de $1,8 \%$ con lo que la tasa resultó tres veces mayor a la población general $\left({ }^{19}\right)$. No se puede afirmar que ello ocurra, ya que el cálculo se basó en un número de casos de AR hallados en una cohorte de pacientes con TBC. Si bien nuestro grupo comparó DI entre una cohorte AR y una de control de una manera retrospectiva, un diseño prospectivo, tendría que contemplar como mínimo 10 años de seguimiento, lo que no daría información oportuna en relación a medidas de control.

El comportamiento de la enfermedad tuberculosa cambia dramáticamente cuando se introduce inhibidores del FNT-alfa, con tasas altas de ET por reactivación. Nuestros pacientes no eran usuarios de terapia biológica. Una reciente teoría epidemiológica sostiene una relación negativa entre AR y TBC en poblaciones americanas $\left({ }^{31}\right)$. Al parecer ciertos cambios genotípicos favorecerían -en este tipo de razas- resistencia a TBC entre los pacientes con AR a lo largo del tiempo $\left.{ }^{(32}\right)$. Uno de estos cambios podría afectar el polimorfismo del FNT-alfa ${ }^{33}$ ) (una citoquina clave en la infección y reactivación del Mycobacterium tuberculoso $\left({ }^{34,35}\right)$, que es fuertemente expresada en AR. Faltan aún más evidencias al respecto. Nuestro estudio ha sido realizado en una población americana con prevalencia alta de TBC y factores diferentes a la disfunción inmunológica per se dada por la enfermedad; o el uso de fármacos inmunosupresores influenciarían el comportamiento del bacilo tuberculoso.

Nuestro análisis no encontró que alguna de las variables analizadas se asociara a riesgo de enfermedad tuberculosa. El contacto epidemiológico intrafamiliar, a diferencia del extrafamilar en nuestro estudio, no representó riesgo, pese a que la mayoría de pacientes procedía de zonas urbanas con gran hacinamiento. Este hecho no es explicado por una adecuada quimioprofilaxis (nuestros 
datos reportan un uso bajo de isoniacida). La mayoría de pacientes con contacto extrafamiliar era trabajador de salud, en quienes el riesgo de desarrollar $\mathrm{TBC}$ es alto $\left({ }^{36,37}\right)$. En relación a los fármacos utilizados como modificadores de enfermedad, grupos europeos han demostrado una incidencia alta de TBC entre pacientes con AR usuarios de FME $\left({ }^{5}\right)$. Nosotros incluimos FME con asociación conocida a TBC o aquellas en las que existe duda sobre su papel $\left({ }^{38}\right)$. Al igual que nuestro grupo, Yoshinaga $\left({ }^{19}\right)$ no encontró asociación de TBC con corticoterapia $\left({ }^{28}\right)$, informada clásicamente como factor de riesgo $\left({ }^{39,40}\right)$. No encontramos además riesgo para uso de MTX. A pesar de tener relevancia en infecciones respiratorias bajas $\left({ }^{41}\right)$, este fármaco muestra controversias en la generación de ET activa $\left({ }^{8}\right)$ y tendría importancia en pacientes infectados con HIV $\left({ }^{42}\right)$, los que no fueron incluidos en nuestro estudio. Comorbilidades como DM e IRCT se asocian a riesgo de TBC $\left({ }^{42,43}\right)$. No se ha encontrado reportes de este análisis entre los pacientes con AR y nuestros hallazgos no encuentran asociación alguna. En nuestro estudio, ninguna comorbilidad se asoció a riesgo. Sin embargo, variables como alcoholismo y la malnutrición no pudieron ser valoradas, dos factores muy asociados al riesgo de TBC. No nos fue posible además evaluar las características de la enfermedad reumatológica en cuanto a la severidad y discapacidad, lo cual podría asociarse a riesgo de TBC. El uso de inmunosupresores en enfermedades crónicas se asocia a enfermedad tuberculosa diseminada y atípica, con elevadas formas extrapulmonares $\left({ }^{44,45}\right)$. El patrón de localización pulmonar o extrapulmonar ofrece resultados disímiles en los estudios realizados entre pacientes con AR (5,17,29, $\left.{ }^{45,46}\right)$. En nuestro estudio, la forma pulmonar fue la más frecuente, a diferencia de lo que ocurre otras EDTC como el LES $\left({ }^{28,30}\right)$.

La SAT recomienda INH en pacientes que reciben crónicamente más de $15 \mathrm{mg} / \mathrm{d}$ de PDN, inmunodeprimidos o en aquellos con un resultado PPD positivo $\left({ }^{47}\right)$. Se ha demostrado el éxito de esta medida en enfermedades reumatológicas $\left({ }^{45,46,48}\right)$. Nosotros encontramos una tasa baja de uso de profilaxis de INH entre nuestros pacientes y la DI de enfermedad tuberculosa en los pacientes AR, respecto a los controles, no mostró diferencia estadísticamente significativa. Esta medida, al parecer, no tendría influencia sobre estos resultados.

La prueba de tuberculina es una de las herramientas más utilizadas para la detección de infección tuberculosa; pero su reactividad entre pacientes con $A R$ es un tema de controversia. El PPD fue negativo en más de $70 \%$ de nuestra cohorte. Un estudio realizado por nuestro grupo demostró que el PPD no era apropiado para detectar la infección entre los pacientes con AR en relación a sujetos controles $\left({ }^{23}\right)$. Se necesita estudios que evalúen métodos seguros de tamizaje entre pacientes AR, para documentar infección tuberculosa.

En conclusión, si bien encontramos una alta incidencia ET entre nuestros pacientes, la condición de tener AR no se comportó como un factor de riesgo independiente para padecerla. Nuestros resultados, sin embargo, alertan sobre la necesidad de vigilar a los pacientes AR como otro grupo de riesgo más para desarrollar TBC y la prioridad de evaluar factores asociados a dicha enfermedad, antes del uso de terapia anti FNT-alfa. Próximos estudios deberían encaminarse a dilucidar si la relación entre AR y el riesgo de padecer tuberculosis obedece a razones de susceptibilidad genética o no y el papel de los mecanismos patogénicos de la enfermedad sobre el comportamiento del bacilo tuberculoso en AR.

\section{AGRADECIMIENTOS}

A las Dras. Graciela S. Alarcón y Cecilia Chung, por sus sugerencias y correcciones en la elaboración de este manuscrito. 


\section{REFERENCIAS BIBLIOGRÁFICAS}

1. Kaipiainen-Seppänen O, Aho K, Nikkarin M. Regional differences in the incidence of rheumatoid arthritis in Finland in 1995. Ann Rheum Dis. 2001;60:128-32.

2. Shichikawa K, Inoue K, Hirota S, Maeda A, Ota H, Kimura $\mathrm{M}$, et al. Changes in the incidence and prevalence of rheumatoid arthritis in Japan, 1965-1996. Ann Rheum Dis. 1999;58:751-6.

3. Gabriel SE. The epidemiology of rheumatoid arthritis. Rheum Dis Clin North Am. 2001;27:269-81.

4. Kalla A. Rheumatoid arthritis in the developing world. Best practice \& Research Clin Rheumatol. 2003;17(5):863-75.

5. Stuck A, Minder C, Frey F. Risk of infectious complications in patients taking glucocorticoids. Rev Infect Dis. 1989;11(6):954-63.

6. Bouza E, Moya JG, Munoz P. Infections in systemic lupus erythematosus and rheumatoid arthritis. Infect Dis Clin North Am. 2001;15(2):335-61.

7. Greenberg SB. Infections in the immunocompromised rheumatologic patient. Critical Care Clinics. 2002;18(4):93156.

8. Doran M, Pond G, Crowson C, O'Fallon W, Gabriel S. Risk of infection in persons with rheumatoid arthritis compared to controls: a population-based study. Arthritis Rheum. 2001;44 Suppl 1:S105.

9. Vadillo C, Hernandez-García C, Pato E, Morado IC, Salido $\mathrm{M}$, Judez E, et al. Incidence and characteristics of tuberculosis in patients with autoimmune rheumatic diseases. Rev Clin Esp. 2003;203(4):178-82.

10. Gomez-Reino J, Carmona L, Rodriguez V, Martin E, Montero D. Treatment of rheumatoid arthritis with tumor necrosis factor inhibitors may predispose to significant increase in tuberculosis risk. Arthritis Rheum. 2003;48(8):2122-6.

11. Keane J, Gherson S, Pharm D, Wise R, Mirabile-Levens E, Kasznica $J$, et al. Tuberculosis is associated with infliximab, a tumor necrosis factor alfa-neutralizing agent. N Eng J Med. 2001;11(345):1098-103.

12. Mohan A, Coté R, Siegel J, Braun M. Infectious complications of biologic treatments of rheumatoid artritis. Curr Opin Rheumatol. 2003;15:179-84.

13. Wolfe F, Michaud K, Anderson J, Urbanisky K. Tuberculosis infection in patients with rheumatoid artritis and the effect of infliximab therapy. Artritis Rheum. 2004;50(2):372-9.

14. Gardam M, Keystone E, Menzies R, Manners S, Skamene E. Anti Tumor necrosis factor agents and tuberculosis risk: Mechanism of action and clinical management. The Lancet infectious disease. 2003;3(3):148-55.

15. European Medicines Agency. The European Agency for the Evaluation of Medicinal Products [monografía en Internet]. London: EMEA; 1995- [citado el 19 de diciembre de 2005]. Disponible en: http://www.emea.eu.int/pdfs/ human/press/pus/

16. Arthritis Drugs Advisory Committee, Food and Drug Administration. Update on the TNF-alfa blocking agents [monografía en Internet]. Rockville, Maryland: Food and Drug Administration; 2003 [citado el 19 de diciembre de 2005]. Disponible en: http://www.fda.gov/ohrms/dockets/ ac/03/briefing/3930b1.htm

17. Carmona L, Hernández-García C, Vadillo C. Increased risk of tuberculosis in patients with rheumatoid arthritis. J Rheumatol. 2003;30(7):1436-9.

18. Wolfe F, Flowers N, Anderson J, Urbansky K. Tuberculosis rates are not increased in rheumatoid arthritis (RA). Arthritis Rheum. 2001;44 Suppl 1:S105.(abstract).

19. Yoshinaga Y, Tatsuya K, Tomoko M, Kagawa H, Yamamura M. Clinical characteristics of Mycobacterium rheumatoid arthritis patients. Mod Rheumatol. 2004;14:143-8.

20. Ministerio de Salud del Perú. Tuberculosis en el Perú. Informe 2000. 1a edición, Lima: MINSA; 2001.

21. Arnett F, Edworthty E, Bloch D, Fries J, Cooper N. The American Rheumatism Association 1987 revised criteria for the classification of rheumatoid artritis. Arthritis Rheum. 1988;31(3):315-23.

22. American Thoracic Society. Tarjetees Tuberculin Testing and treatment of latent tuberculosis infection. Am J Respir Crt Care. Med. 2000;161:1376-95.

23. Ponce de Leon D, Acevedo E, Alfaro J, Sanchez A, Cucho $\mathrm{M}$, et al Attenuated response to purified protein derivative in patients with rheumatoid arthritis: study in a population with a high prevalence of tuberculosis. Ann Rheum Dis. 2005;64:1360-1.

24. Asociación Médica Mundial. Declaración de Helsinki de la Asociación Médica Mundial. Principios éticos para las investigaciones médicas en seres humanos. Adoptada por la $18^{\text {a }}$ Asamblea Médica Mundial Helsinki, Finlandia, Junio 1964, y enmendada por la 52 a Asamblea General Edimburgo, Escocia, Octubre 2000.

25. Zavala D. Situación de la Tuberculosis en el Perú Dirección General de Salud de las Personas. Estrategia Sanitaria Nacional de Prevención y Control de Tuberculosis. Taller TB en Prisiones. Lima, Perú: MINSA; 2004.

26. Fengh Ph, Tan Th. Tuberculosis in patients with systemic lupus erithematosus. Ann Rheum Dis. 1982;41;11-4.

27. Victorio-Navarra T, Arrollo E, Torralba T. Tuberculosis among Filipino patients with systemic systemic lupus eritematosus. Sem Arthritis Rheum. 1996;26:628-3.

28. Tam L, Li E, Wong Sh, Check-Chun S. Risk factors and clinical features among patients with systemic lupus eritematosous in Hong Kong. Scand J Rheumatol. 2002;31:296-300.

29. Yun JE, Lee SW, Kim TH, Jun JB, Jung S, Bae SC, et al. The incidence and clinical characteristics of Mycobacterium tuberculosis infection among systemic lupus erythematosus and rheumatoid arthritis patients in Korea. Clin Exp Rheumatol. 2002;20(2):127-32.

30. Valenzuela G, Sánchez A, Alfaro J, Ponce de León D, Torres J. Acevedo E. Tuberculosis e inmunosupresión. Estudio clínico en pacientes con lupus eritematoso sistémico. Enfermedades del Tórax. 2003;46(2):95-7. 
31. Rothschild B, Rothschild R, Helbling M. Unified theory of the origins of erosive arthritis: Conditioning as a protective/ directing mechanism? J Rheumatol. 2003;30:2095-102.

32. James L. Mobley. Is rheumatoid arthritis a consequence of natural selection for enhanced tuberculosis resistance? Medical Hypotheses. (2004);62:839-43.

33. Correa P, Gomez L, Anaya J. Polymorphism of TNF-alpha in autoimmunity and tuberculosis. Biomedica. 2004;24 Suppl 1:43-51.

34. Ehlers S. Role of tumor necrosis factor (TNF) in host defense against tuberculosis: implications for immunotherapies targeting TNF Ann Rheum Dis. 2003;62(1 Suppl II):ii37ii42

35. Mohan V, Scanga Ch, Yu K, Scout H, Tanaka K, Tsang E, et al. Effects of tumor factor alpha on host immune response in chronic tuberculosis: posible role for limiting pathology. Infection and Immunity. 2001;69(3):1847-55.

36. Rey de Castro J, Carcelén A. Infección y enfermedad tuberculosa en trabajadores de un Centro de Salud. Rev Med Hered. 1992;3(1):19-21.

37. Bonifacio L, Saito M, Gilman M, Leung F, Chavez N, et al. High risk for tuberculosis in hospital physicians, Peru. Emerging Infectious Dis. 2002;8(7):747-8.

38. Cunnane G., Doran M. Infections and biological therapy in rheumatoid arthritis Best Practice \& Research Clinical Rheumatol. 2003;17(2):345-63.

39. Agrawal PN, Gupta D, Aggarwal AN, Behera D. Incidence of tuberculosis among patients receiving treatment with oral corticosteroids. Assoc Physicians India. 2000;48(9):881-4.

40. Kim HA, Yoo CD, Baek HJ, Lee EB, Ahn C, Han JS, et al. Mycobacterium tuberculosis infection in a corticosteroidtreated rheumatic disease patient population. Clin Exp Rheumatol. 1998;16(1):913.

41. Van der Ven M, Van der Heide A, Bijlsma J. Infection rate and use of antibiotics in patients with rheumatoid arthritis treated with methotrexate. Ann Rheum Dis. 1994;53:224-8.
42. Hussein M, Mooij M, Haysam R. Tuberculosis and chronic disease. Seminars in Dialysis. 2003;16(1):38-51.

43. Doran M, Crowson S, Pond G, O Fallon M. Predictors of infection in rheumatoid arthritis. Arthritis Rheumat. 2002;46(9):2294-300

44. Skogber K, Riitu P, Tukiainen P, Valtonen V. Effect of immunosuppresive on the clinical presentation and outcome of tuberculosis. Clin Inf Dis. 1993;17:1012-7.

45. Hernandez-Cruz B, Cardiel M, Villa A, Alcocer-Varela J. Development, recurrence, and severity of infections in Mexican patients with RA. A nested case-control study. J Rheumatol. 1998;25(5):1900.

46. Tajabayashi K, Hanaoka H. Collagen diseases with opportunistic infections. Nihon Rinsho Meneki Gakkai Kaishi. 2004;27(3):156-63.

47. American Thoracic Society. Diagnostic standards and classifications of tuberculosis in adults and children. Am J Respir Crit Care Med. 2000;161:S221-S247.

48. Gaitonde S, Pathan E, Sule A, Mitta G, Joshi R. Efficacy of isoniazid prophylaxis in patients with systemic lupus erythematosus receiving long term steroid treatment. Annals Rheumatic Dis. 2002;61:251-3.

Manuscrito recibido el 25 de setiembre de 2006 y aceptado para publicación el 16 de octubre de 2006.

\section{Correspondencia:}

Dra. Rocío Gamboa Cárdenas

Calle Tungasuca 409. San Luis

Lima 30, Perú.

Correo-e:rvgc1@yahoo.com 